\title{
DURIAN RECOGNITION BASED ON MULTIPLE FEATURES AND LINEAR DISCRIMINANT ANALYSIS
}

\author{
Mas Rina Mustaffa ${ }^{1}$, Nyon Xin $\mathbf{Y i}^{2}$, Lili Nurliyana Abdullah ${ }^{3}$, and Nurul Amelina Nasharuddin ${ }^{4}$ \\ ${ }^{1,2,3,4}$ Department of Multimedia, Faculty of Computer Science and Information Technology, \\ Universiti Putra Malaysia 43400 UPM Serdang, \\ Selangor Darul Ehsan, MALAYSIA
}

Email: MasRina@upm.edu.myํㅣㄱ.liyana@upm.edu.my²,nurulamelina@upm.edu.my ${ }^{3}$

DOI: https://doi.org/10.22452/mjcs.sp2018no1.5

\begin{abstract}
Many fruit recognition approaches today are designed to classify different type of fruits but there is little effort being done for content-based fruit recognition specifically focuses on durian species. Durian, known as the king of tropical fruits, have few similar characteristics between different species where the skin have almost the same colour from green to yellowish brown with just slightly different shape and pattern of thorns. Therefore, it is hard to differentiate them with the current methods. It would be valuable to have an automated content-based recognition framework that can automatically represent and recognise a durian species given a durian image as the input. Therefore, this work aims to contribute to a new representation method based on multiple features for effective durian recognition. Two features based on shape and texture is considered in this work. Simple shape signatures which include area, perimeter, and circularity are used to determine the shape of the fruit durian and its base while the texture of the fruit is constructed based on Local Binary Pattern. We extracted these features from 240 durian images and trained this proposed method using few classifiers. Based on 10-fold cross validation, it is found that Logistic Regression, Gaussian Naïve Bayesian, and Linear Discriminant Analysis classifiers performed equally well with $100 \%$ achievement of accuracy, precision, recall, and F1-score. We further tested the proposed algorithm on larger dataset which consisted of 42337 fruit images (64 various categories). Experimental results based on larger and more general dataset have shown that the proposed multiple features trained on Linear Discriminant Analysis classifier able to achieve $72.38 \%$ accuracy, $73 \%$ precision, $72 \%$ recall, and $72 \% \mathrm{~F} 1$-score.
\end{abstract}

Keywords: Multiple features, Colour, Shape, Texture, Durian recognition

\subsection{INTRODUCTION}

Image recognition is a process of identifying an object in a digital image. There are many recognition systems existed such as for face [1-2], voice [3], handwriting [4], and others [5-7].

Malaysia is a country rich in choices of tropical fruits. All the local fruits are available all year round in the market but some are seasonal. For example, durian (Durio zibethinus) is the 'king of fruits' which can only be found during December to January and June to August and Malaysia is the largest exporter for durian after Thailand [8-9]. All the varieties of durian are hard-shelled with green thorns and they are hard to differentiate. Experienced farmer in the agriculture domain may find it easy to categorise various durian species but it would be extremely hard for others to identify and classify durian species manually. It is important for a person to have some knowledge in recognising various durian species as the unit price is relatively high when compared to other tropical fruits.

Two important steps in an image recognition framework are the feature extraction and its classifier. Feature extraction is very important where the right features will lead to an accurate representation and recognition, and vice versa [10]. Although the fusion of features is important in expressing the different and useful information of an image, at the same time, the complexity of an algorithm has to be considered. Feature extraction methods are domain specific where a method may give good representation for one domain but performs badly for other domains. Classifier on the other hand is important in classifying the images based on the extracted features. Selecting a good classifier will produce a good classification model for the recognition framework as a whole. There are two approaches in image classification, which are supervised and unsupervised classification [11]. Supervised classification requires training data to infer the 
classifier, which will then be applied to the test or actual sets of images. Unlike supervised classification, unsupervised classification does not rely on a training data, instead it uses clustering techniques which measure the distance between images and group the images with common features together.

Fruit recognition system uses fruit image's content for analysis, identification, and classification in the agriculture domain. Automatic fruit recognition system using computer vision is a useful tool in a variety of fields, such as education, image retrieval, and agricultural research [12]. To our knowledge, only few researches have been done on content-based recognition specifically for durian species. Therefore, this research aims to introduce a feature fusion extraction method which can effectively describe and represent four different types of durian, namely D24, D101, D160, and D197. In order to differentiate the various types of durian, the pattern of the fruit's base, spines on the durian skin, and the fruit's shape will be utilised. For example, D24 has smaller base size with thin spines, D101 has ellipsoid shape with long and thin spines, D160 has the shape of a heart with short and thick spines, while D197 has a distinct star-shaped base. A classification model will then be generated based on the extracted features.

The outline of this paper is as follows. Section 2 provides the discussion on related works. The proposed framework is described in Section 3. Section 4 explains the framework for evaluation while analysis of results is described in Section 5. Finally, the conclusion and future direction are presented in Section 6.

\subsection{LITERATURE REVIEW}

Fruits representation and recognition are crucial and can be a considered a very challenging task. Based on several literature reviews, we have identified few existing feature extraction and recognition approaches for fruits.

Plant Variety Protection Malaysia [13] is a form of intellectual property right of plant varieties in Malaysia. In Malaysia, all of the durian varieties registered with the Department of Agriculture (DOA) since year 1993 is recorded in Varieties Registered for National Crop List. This online database of registered durian varieties in Malaysia provides the list of details and image link. Users can search durian species by providing input in the form of a keyword such as variety registration number which usually formed by alphabet $\mathrm{D}$ and followed by unique number. Besides, users can go through the entire list in the database to search the durian species. The advantage of this database is providing trustable sources of durian information for user. The limitation of this database is it only allows the search of durian species using keyword. In this case, users who do not know the name of the durian species only can key in "durian" and get a long-list result. Users cannot search the details of durian species directly and have to go through all of the results one by one. This method will consume a lot of time to search for an exact durian species. Moreover, the images of durian species are not available for all species. Users cannot imagine how exactly the particular species look like by only through the text description.

The authors in [14] classify durian varieties based on vocabularies of durian characteristics and image annotations using conceptual graph method. Durian varieties can be determined semantically, however, the work is done semiautomatically and various characteristics of a durian species need to be determined first before classification or recognition can take place.

Based on [13] and [14], it can be observed that some efforts have been done to categorise and manage various durian species. However, all of the above approaches are still based on text. Often, we are in a situation where we may have visual information of an item but without any other textual description. Hence, it would be beneficial to have an automated content-based recognition framework that can automatically represent and recognise a durian species given a durian image as the input.

Previously, there are few existing works on content-based fruit recognition. Automatic fruit recognition system using computer vision is a useful tool in a variety of fields, such as education, image retrieval, and agricultural research [15]. Fruit recognition uses fruit image's content for analysis, identification, and classification in the agriculture domain. Some existing works such as recognition of various fruits [16-21] or even focusing on specific fruits such as olive [22], persimmon [23], almond [24], apple [25], papaya [26], and durian [27-28].

Authors in [16] proposed a fruit recognition system using combination of colour, shape, texture and size-based features to perform classification of fruit images. Color details (RGB) is extracted as colour descriptor. The area and perimeter are calculated to obtain the roundness value which will act as the shape feature. Texture on the other hand is represented by computing the entropy value of the image. Lastly, the K-Nearest Neighbour (KNN) algorithm will figure out the closest class label of fruit image to the input image. The dataset used is 36 fruit images. This system 
achieves up to $95 \%$ of accuracy. The advantage of this system is it is invariant to rotation and translation. However, the proposed method leads to high computation time as quite a number of features are utilised.

Authors in [17] represent fruit images based on few shape features such as area perimeter of the fruit region, major axis length, minor axis length and distance between the foci of an equivalent ellipse, width, height and area of minimum bounding box, and area and perimeter of smallest convex polygon. These shape features allow for the image representation to be invariant to translation, rotation, uniform scaling, surface features, and growth stage of fruit. Three classifiers which are Naive Bayes (NB), Neural Network (NN), and K-NN are experimented to determine the suitable classifier. For the dataset, 210 images of seven fruit classes are either captured using a smartphone camera or downloaded from websites. Each class contains 30 images. Based on the conducted experiment, it has been shown that a better recognition rate of between $88-95 \%$ is achieved when using NB classifier instead of NN or K-NN.

Authors in [18] combine Zoning and Character-Edge Distance methods together with Discrete Fourier Transform as the feature descriptors for fruit image representation. This feature representation is fed into two classifiers, Multilayer Perceptron and K-NN. It has been observed that Multilayer Perceptron performed better classification and able to obtain a classification rate of $97.5 \%$ when experimented on 163 fruit images.

Authors in [22] focus on a specific fruit which is olive by analysing the RGB images to determine the size and weight of the olive fruits. Morphological analysis is also conducted to determine any overlapping division. Feedforward NN with backpropagation learning algorithm is utilised as the classifier. The dataset size is not stated in the paper. The recognition accuracy reported is $97 \%$.

Authors in [23] focus on the recognition of 50 persimmon fruits based on Fourier transform near infrared (FT-NIR) spectrometry information as its representation and Least squares SVM as the classifier. 98\% classification rate has been obtained.

Authors in [24] utilised the Bag-of-Feature $(\mathrm{BoF})$ model for almond feature representation where they experimented with few keypoint detectors such as Harris, Harris-Laplace, Hessian, Hessian-Laplace, and Maximally Stable External Regions (MSER) together with Scale Invariant Feature Transform (SIFT). K-means clustering is then used to build the codebook from keypoint descriptors. Various codebook size is also being experimented. Three classifiers were tested on 2000 sweet and bitter almonds (K-NN, Linear SVM, and Chi-square SVM) which were randomly sampled from an almond orchard in a commercial farm in Yazd, Iran. It has been observed that the Harris-Laplace combined with SIFT descriptor, with a codebook size of 500, and Chi-SVM provides a reasonable recognition rate of $97.5 \%$ for sweet almonds and $95.5 \%$ for bitter almonds.

Authors in [25] performed apple identification by first performing image enhancement based on hommomorphic filtering for light compensation. The RGB colour differences in the RGB colour space are then computed. Image segmentation is then performed based on simplified pulse coupled neural network (PCNN) based on minimum cross entropy followed by noise removal using morphological operations. An Improved Hough transform is utilised to detect the circularity feature of the apple image according to the edge obtained by smallest univalues segment assimilating nucleus (SUSAN) edge detection algorithm. The shape of a ripe apple is similar to a circle, where this feature will be used as the final apple image representation. 50 apple images are used for testing and the recognition rate obtained is $93 \%$.

Papaya grading is done by [26] where the authors experimented on 21 hand-crafted colour features obtained from three colour spaces (mean of R, G and B, normalised mean of R, G and B, area of R, G and B, normalised area of R, $\mathrm{G}$ and $\mathrm{B}$, normalised differential index of red to green, red to blue and green to blue, mean value of $\mathrm{H}, \mathrm{S}$ and $\mathrm{V}$, and mean of $\mathrm{L}, \mathrm{a}$ and $\mathrm{b}$ ). These colour information is extracted from the papaya's skin. It has been found that the normalised mean of the separate R, G, and B channels provide the best colour representation for the papaya images. Random forest classifier is used to determine the stage of ripeness. Before feature extraction is carried out, some pre-processing steps were done which include region of interest (ROI) detection and normalisation. In order to create the dataset, 57 papaya fruits were purchased in a retail market and two colour images are obtained from each papaya. The classification rate obtained is $78.1 \%$.

There are also some works on fruit recognition which applied the deep learning approaches. The utilisation of deep learning approaches, for example Convolutional Neural Network (CNN), over traditional machine learning methods allow for automatic representation of the fruits rather than the needs to perform hand-crafted feature extraction. Authors in [19] use Faster Region-based Convolutional Neural Network (Faster R-CNN) for colour and near-infrared 
fruit images instead of the single CNN. Images of sweet pepper (122), rock melon (135), apple (64), avocado (54), mango (170), and orange (57) are utilised as the dataset. F1-score of 0.838 is reported.

Author in [20] uses bottom-up graph-based visual saliency (GBVS) model to determine the significant area in an input image and Visual Geometry Group model for feature representation and classification. The image dataset consists of 12173 grouped into 26 fruits and vegetables categories. A recognition rate of $95.6 \%$ is reported.

Authors in [21] on the other hand perform fruit recognition based on the CNN. Using selective search algorithm, fruit regions of an image are extracted, which are then selected based on the entropy values. The selected regions will become the input to the CNN. 5330 of fruit images is used as the dataset. The authors have contributed to a recognition rate of $99.77 \%$.

In regards to content-based recognition focusing on durian domain, we managed to find only two related works. Authors in [27] recognise two types of durian (Chanee and Monthong) by analysing the peak of the fruit's thorns and its geometric outlines. 40 Durian images are used for evaluation. An accuracy of $94.44 \%$ has been obtained.

The focus of authors in [28] is however rather different. Although it is a work on content-based recognition for durian, their aim is on differentiating between unripe and ripe durians based on the spectral features of striking sound instead of classifying durian according to its species.

From reviewing few existing fruit recognition works, it can be concluded that image processing is required prior to feature extraction. For feature extraction, the most suitable feature descriptor has to be constructed based on the targeted domain since feature extraction methods are domain specific. The works done by [16-18] [22-26] show that the feature descriptors used are different and unique based on the respective fruit. The feature descriptor will also need to be invariant to rotation and translation or illumination to increase the robustness of the proposed work. From the literature review and to our knowledge, there is very little exploration being done for content-based durian species recognition [27-28]. For durian species images, suitable feature representations will also need to be determined. With 133 of durian varieties [13], it will be a challenging task to differentiate all durians since they look approximately the same. However, shape and texture features would most probably be beneficial for representation since different species will have slightly different shape of the fruit's base and pattern of spines on the durian skin.

Lastly, it is very important to experiment on the classifier so that the most suitable classification model can be generated to provide a positive recognition rate for the proposed recognition framework. From the literature, we found that some of the commonly used classifiers for fruit recognition are NB and SVM. Deep learning approaches adopted in [19-21] is relatively good however, it is known for its need of a very large amount of data in order to provide good recognition rate. This may not be suitable for durian species or agriculture since not many datasets are available publically. In most cases, researchers will need to gather their own data which is very time consuming to obtain a very large amount of data. Traditional machine learning approaches can perform equally well with small dataset. It is also not very computationally expensive for training as compared to deep learning approaches. Therefore, NB, SVM and together with few other traditional machine learning approaches will be analysed and studied further to determine the most suitable classification model for the proposed durian images.

Therefore, this research aims to introduce a feature extraction method which can effectively describe and represent durian images. Due to the exclusive characteristics being extracted by different methods, there is still a need to construct a feature extraction technique based on multiple features. In our previous paper, the shape descriptor for durian representation has been constructed. It provides great result. We are extending the work done in [29] to include texture feature so that more effective method can be developed for durian representation. A classification model will then be generated based on the extracted features.

\subsection{PROPOSED METHOD BASED ON MULTIPLE FEATURES}

Fig. 1 below shows the complete steps of the proposed method based on two multiple features. The novelty of this framework is based on the combination of the feature extraction methods used to accurately represent the respective shape and texture information of durian images as well as the suitable classification model. 


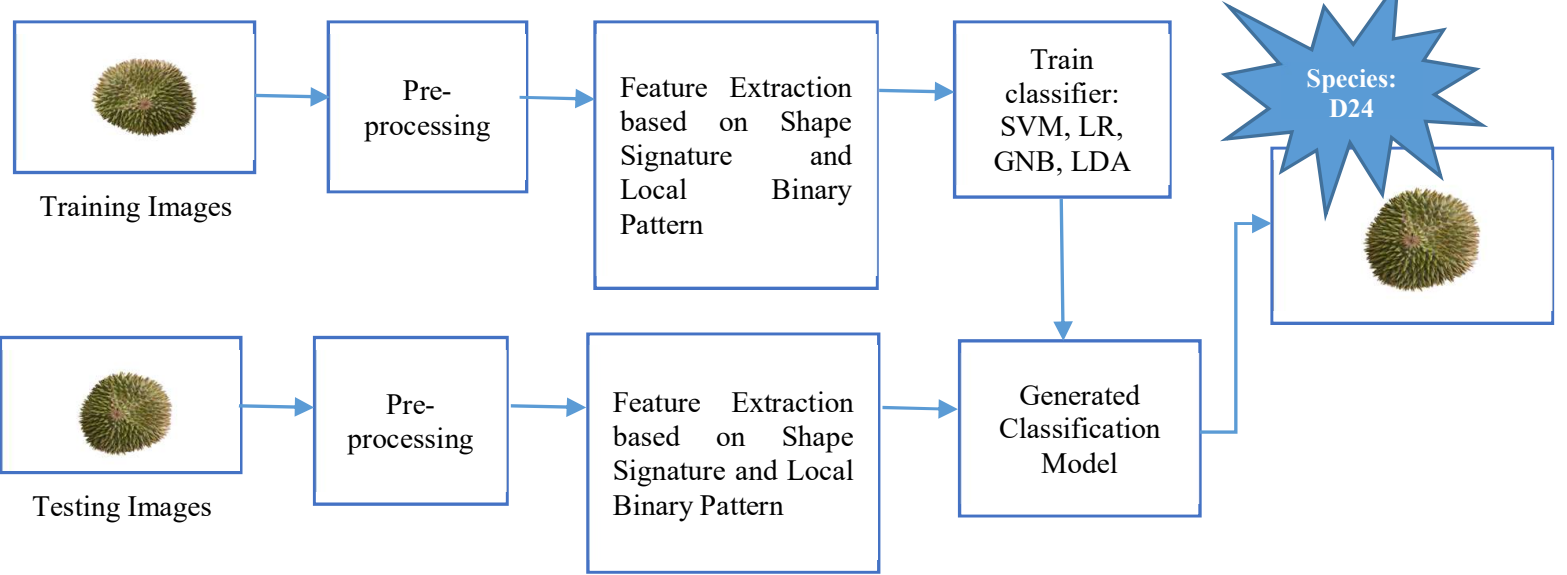

Fig. 1: Feature extraction based on Shape Signature and Local Binary Pattern

Each of the components in Fig. 1 will be explained in the following sub-sections.

\subsection{Image Pre-processing}

First of all, for ease of computation and management, we have resized all images to a standard size of $64 \times 64$. This is because the images in the dataset come in various sizes and some may be too large to be processed efficiently.

\subsection{Shape Feature Extraction based on Shape Signature}

The shape signature was adopted from our previous work [29]. However, some changes have been made to the method where we have further reduced the image size from $320 \times 240$ to $64 \times 64$ as we have found out through a conducted experiment that this is a sufficient size for representation and able to reduce computation time. Instead of utilising the HSV colour model, we have converted the image into grayscale as this is found to be better representation rather than converting from HSV colour model to binary image. The noises in the image are then removed using morphological operations. The first morphological step is 'opening' on the binary image with a square structuring element radius of two. Then, the eight connected objects which are lesser than 200 pixels are removed from the binary image.

Next, global contour-based and region-based shape features are extracted. The area and perimeter of region of interest are obtained. Area captures the number of pixels within a shape while perimeter extracted the number of pixels in the boundary of an object. From the calculated area and perimeter of a shape, the durian base circularity value can be calculated using Equation (1) below:

$$
\text { Circularity }=4 \pi\left(\frac{\text { Area }}{\text { Perimeter }}\right)
$$

Circularity is the ratio of the area of a shape to the area of a circle with the same convex perimeter. A circularity value of one indicates a perfect circle.

\subsection{Texture Feature Extraction Based on Local Binary Pattern}

Local Binary Pattern (LBP) is then computed on a grayscale image. This method captures the local texture information of an image and its strength by analysing the relationship between pixels with its immediate neighbourhood. The original $3 \times 3$ neighbourhood is thresholded by the center value. The thresholded neighbourhood pixels values are then multiplied by the weights given to the corresponding pixels. Finally, the values of the eight pixels are summed to obtain the number of the texture unit. There is a possibility of $2^{8}=256$ texture units. The LBP can be computed based on the following Equation (2). 


$$
L B P_{R, P}=\sum_{p=0}^{P-1} s\left(g_{p}-g_{c}\right) \cdot 2^{p},
$$

where $g_{p}$ is the neighbourhood pixels, $g_{c}$ is the center pixel value, $p$ is the sampling point, and $R$ is the radius. An execution of the LBP descriptor is shown in Fig. 2 below.

\begin{tabular}{|c|c|c|}
\hline 3 & 7 & 2 \\
\hline 8 & 4 & 1 \\
\hline 2 & 3 & 5 \\
\hline
\end{tabular}

Neighbourhood of a grayscale image

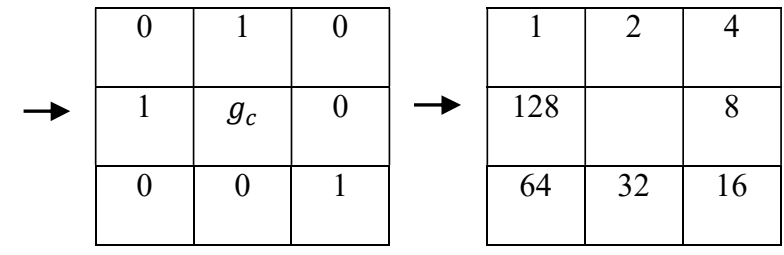

Binary code for $\geq g_{c}$

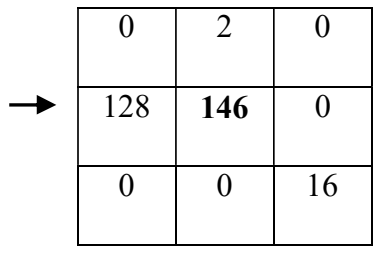

Generated LBP: 146

Fig. 2: Obtaining LBP for $3 \times 3$ neighbourhood

LBP is considered to be a suitable texture descriptor due to its high discriminative power, simple and efficient computation, invariant to grayscale changes, and robust to monotonic illumination change.

\subsection{Feature Fusion Vector}

All of these generated feature vectors will then be combined to represent an image. In total, there are three shape features (area, perimeter, and circularity), and 10 LBP texture features.

\subsection{Classification}

Several classification models exist which are widely used to solve many real-world recognition problems including for fruits recognition. There is no one classification model that suits for all problems. Hence, we have tested few classifiers for this work which include Support Vector Machine (SVM), Logistic Regression (LR), Gaussian Naïve Bayesian (GNB), and Linear Discriminant Analysis (LDA).

SVM classifier is created by Corinna Cortes and Vladimir Vapik [30]. It can be used for classification task as well as for regression task. The SVM works well in a high-dimensional space by capitalising the margin between classes through the generation of hyperplanes. One prominent advantage of SVM is that non-linear classification can be done effectively by means of kernel-trick function, where the classifier can be constructed without the feature space [31]. It is also good at generalisation, resulted in less overfitting, robust, works when training examples comprise errors, and fast evaluation of the learned target function.

LR classifier builds a target model through independent features [32]. It is widely used for forecasting problems and determining the cause and effect of relationship between variables. This classifier provides information about how relevant a feature is and its direction of association (positive or negative). LR is very robust to noise, efficient, and can overcome overfitting. It is also not necessary to have equal variance in each group or the independent variables not necessarily to be normally distributed.

GNB is based on conditional probabilities [33]. It uses Bayes' theorem that calculates a probability by counting the occurrence of values and combinations of values in the historical data. GNB can learn the association between features, simple, fast computation, highly scalable, can make probabilistic predictions, not sensitive to irrelevant features, and easily trained even on small dataset.

LDA separates objects in two or more groups based on Mahalanobis metric or generalised distance according to linear combination of features [34]. It also gives the same linear separating decision surface as Bayesian maximum likelihood 
discrimination in the case of equal class covariance matrices. LDA works well for small samples with many variables (robustness to non-normality and even to mildly variation in covariance matrices), simple, and fast.

\subsection{EXPERIMENTAL SETUP}

This research is conducted based on the quantitative experimental approach. According to Leedy and Ormrod [35], experimental study is done to investigate the possible influences that one factor or condition may have on another factor or condition. This approach is suitable for this study since the main focus of this research is to examine the possible influences that the proposed method has in increasing the recognition performance compared to the existing comparable methods.

There are two experiments being conducted for this work. Experiment one is to determine the classifier that is suitable for durian image dataset.

Experiment two is to evaluate the proposed method on larger and more general dataset, which is a fruit image dataset of many varieties.

\subsection{Hardware and Software Specifications}

All of the experiments mentioned in this work have been conducted on a desktop running on Microsoft Windows 7 Professional operating system, with Intel ${ }^{\circledR}$ Core $^{\mathrm{TM}}$ i7-4700MQ CPU, 2.40GHz, and 32 GB Random Access Memory. MATLAB R2018a is used for feature extraction while Python is utilised for executing the image recognition.

\subsection{Dataset Collection}

There are two image datasets utilised for this work. For the first experiment, 240 durian images are used to determine the most suitable classification model for the proposed feature fusion method. In our previous work [29], only 40 durian images have been used. For this experiment, we have managed to expand the collection. There are four different types of durian, D24, D101, D160, and D197. There are 60 images for each species. We separated the dataset to $70 \%$ for training and $30 \%$ for testing. 10 -fold cross validation is used for evaluation. Sample of the mentioned dataset can be found in Fig. 3 below.
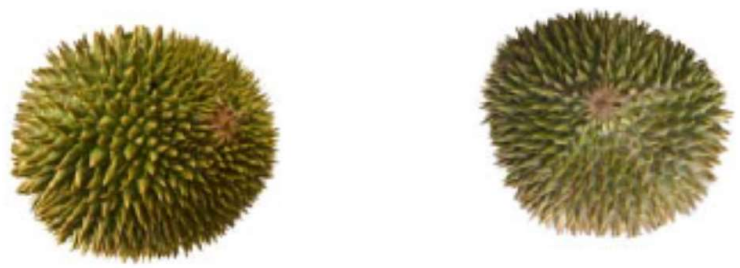

(a)
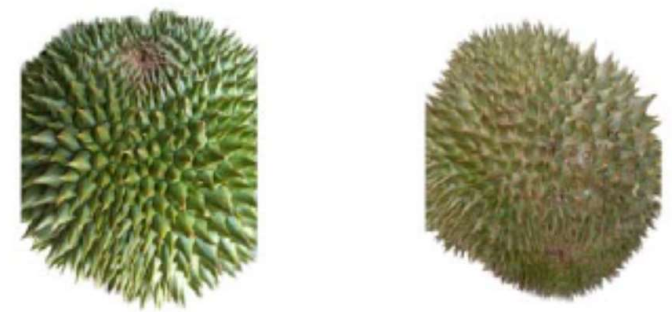

(c)

Fig. 3: Samples of the durian dataset (a) D24 (b) D160 (c) D101 (d) D197

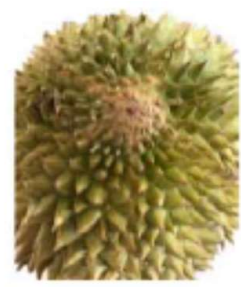

(b)

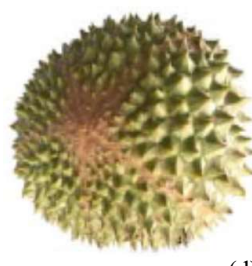

(d)

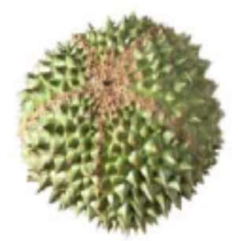


It is rather a challenging task to obtain a larger set of the durian collection. To our knowledge, there is no existing dataset on durian species images. Therefore, these durian images have been collected manually. However, the dataset collection can only be done during the durian season where we approached few durian orchards in Raub, Pahang. The labelling of durians for the ground truth are assisted by the owners and staff of the durian orchards who have had years of experience with durian and can determine durian species. Pre-processing is done to the images when necessary to obtain a reasonably good quality of digital images.

Apart from evaluating the proposed work on durian images, we also conducted testing on larger dataset. Since we only managed to collect 240 durian images, the proposed feature fusion method is tested on Fruits-360 dataset which contains 42337 fruits images of 64 categories [36]. This dataset also contains different varieties of the same fruit which are treated as belonging to different classes (e.g. for apple alone, there are 10 classes). A good feature extraction method and classifier should be able to differentiate these apple varieties.

The number of images for each category varies from one to another and they vary significantly in terms of position, orientation, and size. All images are utilised for this experiment where $70 \%$ is used for training and $30 \%$ is used for testing. We have conducted the 10 -fold cross validation for evaluation. The dataset can be downloaded from https://www.kaggle.com/moltean/fruits. Fig. 4 shows few samples of the Fruits-360 dataset.
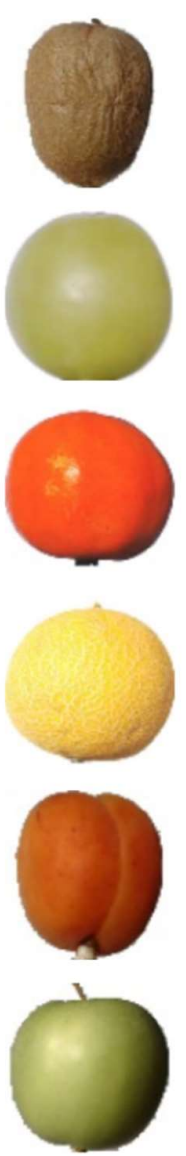
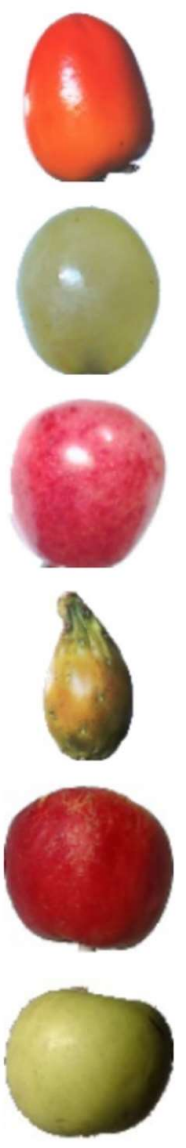
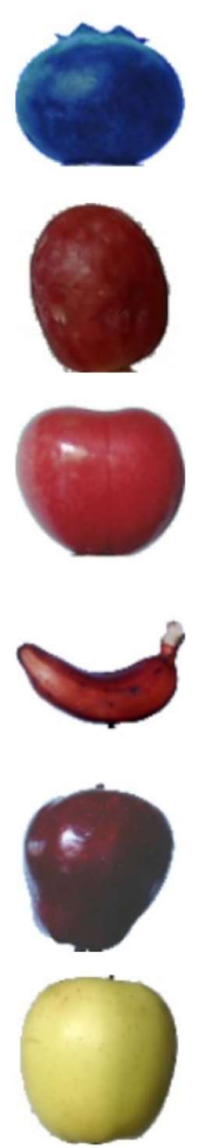
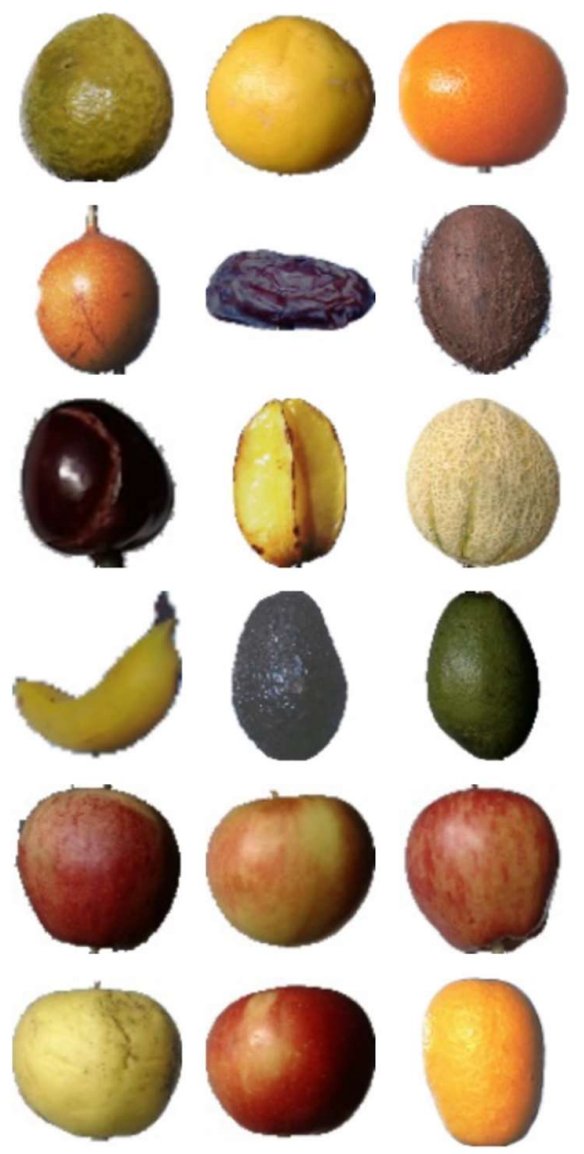


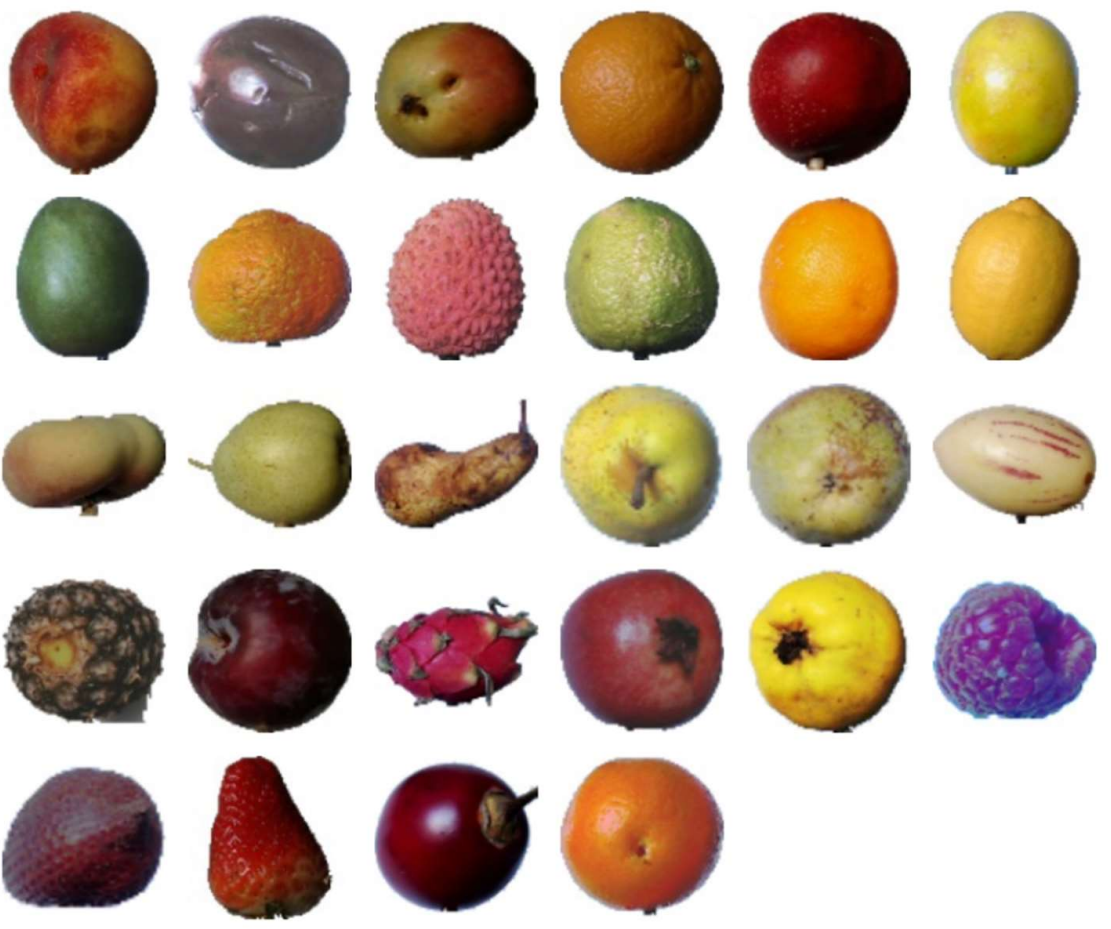

Fig. 4: Samples from Fruits-360 image dataset [36]

\subsection{Evaluation Measurement}

The proposed method will be compared with the benchmark methods using various recognition performance measurements explained below. These measurements are known for evaluating performance of classification framework. Explanation on the evaluation measurements are based on the following confusion matrix as shown in Fig. 5 below. A confusion matrix is a table that is often used to describe the performance of a classification model on a set of test data for which the true values are known. True positives and true negatives are the observations that are correctly predicted while the false positives and false negatives are which we would want to minimise.

\section{Predicted}

\begin{tabular}{|c|c|c|c|}
\hline \multirow{3}{*}{ Actual } & & Negative & Positive \\
\hline & Negative & True Negative & False Positive \\
\hline & Positive & False Negative & True Positive \\
\hline
\end{tabular}

Fig. 5: Confusion matrix

Accuracy is the ratio of correctly predicted observation to the total observations. Accuracy can be calculated based on the Equation (3) below.

$$
\text { Accuracy }=\frac{\text { True Positive }+ \text { True Negative }}{(\text { True Positive }+ \text { False Positive }+ \text { Fal } \quad \text { Negative }+ \text { Tru } \quad \text { Negative })}
$$

The precision and recall measures are one of the widely used due to its simple calculations and results obtained are easily interpreted. A precision rate determines how precise or accurate the model out of those predicted positive. The equation is as shown in Equation (4) below. 


$$
\text { Precision }=\frac{\text { True Positive }}{\text { True Positive False Positive }}
$$

A recall rate is defined as the determination of how many of the Actual Positives the model capture through labelling it as Positive (True Positive). The equation is as shown in Equation (5) below.

$$
\text { Recall }=\frac{\text { True Positive }}{\text { True Positive }+ \text { False Negative }}
$$

F1-score on the other hand is the weighted average of precision and recall. The F1-score can be obtained based on the Equation (6) below.

$$
F 1=2 \times \frac{\text { Precision } \times \text { Recall }}{\text { Precision }+ \text { Recall }}
$$

\subsection{RESULTS AND DISCUSSION}

Two experiments are conducted in this work. For the first experiments, the proposed colour-shape descriptor is evaluated using 240 durian images. In the second experiment, larger dataset which consists of 42337 fruit images is utilised. For both experiments, several classifiers are tested based on several performance measurements.

\subsection{Classification Performance based on Durian Image Dataset}

In this experiment, we feed the shape-only feature, texture-only feature, and shape-texture feature to few classifiers which include Support Vector Machine (SVM), Logistic Regression (LR), Gaussian Naïve Bayesian (GNB), and Linear Discriminant Analysis (LDA). These classifiers are chosen based on the characteristics as mentioned in Section 3.6. 240 durian images of four categories (D101, D160, D197, and D24) are used as the dataset. We split the dataset to $70 \%$ for training (160 images) and $30 \%$ for testing (72 images). Each predictor is trained using 10-fold cross validation. Several performance measurements are captured such as the accuracy (A), precision (P), recall (R) and F1score (F1). The experimental results obtained for the respective shape-only feature, texture-only feature, and feature fusion is as shown in Tables $1-4$ below.

Based on the results in Table 1, it can be observed that the proposed shape-texture descriptor has achieved the best result with an accuracy score of $100 \%$ when using GNB and LDA classifiers. Slightly lower accuracy rates are obtained when utilising SVM and LR classifiers. However, the accuracy of the proposed shape-texture descriptor is much higher in comparison to the shape-only and texture-only descriptors. Shape-only descriptor on the other hand performed better than texture-only descriptor. This is because the shape of the durian's base is more important in distinguishing the various types of durian species in comparison to the texture of the durian's skin. However using shape or texture feature alone will not return the best accuracy rate. The combination of these two features which consider both the shape of the durian's base and the texture of the durian's skin that contributed to the best accuracy. It can be observed as well that this multiple feature descriptor performed at its best based on GNB or LDA classifier.

Table 1: Accuracy for single feature and combined features based on various classifiers

\begin{tabular}{|c|c|c|c|c|}
\hline Method / Classifier & SVM (\%) & LR (\%) & GNB (\%) & LDA (\%) \\
\hline Shape-only [29] & 95.83 & 70.83 & 97.22 & 88.88 \\
\hline Texture-only & 20.83 & 63.88 & 86.11 & 94.44 \\
\hline Shape-Texture (proposed) & 95.83 & 79.16 & 100 & 100 \\
\hline
\end{tabular}

Tables $2-4$ show the precision, recall, and F1-score for the respective shape-only descriptor, texture-only descriptor, and shape-texture descriptor. We have captured these various performance measurements according to the four durian species. Based on our observation, it can be concluded that in general, feature fusion descriptor performed the best, 
followed by shape-only descriptor and then texture-only descriptor. Among the durian species, some of the classifiers had difficulties in classifying D101 and D160 correctly. This is probably due to the reason that the durian's base and the durian's skin of these two different species are almost similar. It can also be observed that different performance is obtained when using different measurement. Precision is a good measure to determine when the costs of False Positive is high, recall can visualise when there is a high cost associated with False Negative, and F1-score is needed when a balance between precision and recall needs to be observed. Hence, from the tables, it is shown that featurefusion descriptor obtained a very consistent and high performance where it able to achieve $100 \%$ precision, $100 \%$ recall and 100\% F1-score for GNB and LDA classifiers.

Table 2: Precision, Recall, and F1-score for shape feature according to each durian species and classifiers

\begin{tabular}{|c|c|c|c|c|c|c|c|c|c|c|c|c|}
\hline \multirow{2}{*}{$\begin{array}{l}\text { Species / } \\
\text { Classifier }\end{array}$} & \multicolumn{3}{|c|}{ SVM (\%) } & \multicolumn{3}{|c|}{ LR (\%) } & \multicolumn{3}{|c|}{ GNB (\%) } & \multicolumn{3}{|c|}{ LDA (\%) } \\
\hline & $\mathbf{P}$ & $\mathbf{R}$ & F1 & $\mathbf{P}$ & $\mathbf{R}$ & F1 & $\mathbf{P}$ & $\mathbf{R}$ & F1 & $\mathbf{P}$ & $\mathbf{R}$ & F1 \\
\hline D101 & 100 & 84 & 100 & 47 & 100 & 64 & 90 & 100 & 95 & 87 & 68 & 76 \\
\hline D160 & 88 & 100 & 93 & 0 & 0 & 0 & 100 & 90 & 95 & 76 & 90 & 83 \\
\hline D197 & 100 & 100 & 100 & 100 & 100 & 100 & 100 & 100 & 100 & 100 & 100 & 100 \\
\hline D24 & 100 & 100 & 100 & 100 & 100 & 100 & 100 & 100 & 100 & 100 & 100 & 100 \\
\hline Average & 96 & 96 & 96 & 57 & 71 & 61 & 97 & 97 & 97 & 89 & 89 & 89 \\
\hline
\end{tabular}

Table 3: Precision, Recall, and F1-score for texture feature according to each durian species and classifiers

\begin{tabular}{|c|c|c|c|c|c|c|c|c|c|c|c|c|}
\hline \multirow{2}{*}{$\begin{array}{l}\text { Species / } \\
\text { Classifier }\end{array}$} & \multicolumn{3}{|c|}{ SVM (\%) } & \multicolumn{3}{|c|}{ LR (\%) } & \multicolumn{3}{|c|}{ GNB (\%) } & \multicolumn{3}{|c|}{ LDA (\%) } \\
\hline & $\mathbf{P}$ & $\mathbf{R}$ & F1 & $\mathbf{P}$ & $\mathbf{P}$ & $\mathbf{R}$ & F1 & $\mathbf{P}$ & $\mathbf{P}$ & $\mathbf{R}$ & F1 & $\mathbf{P}$ \\
\hline D101 & 0 & 0 & 0 & 68 & 100 & 81 & 100 & 100 & 100 & 100 & 79 & 88 \\
\hline D160 & 0 & 0 & 0 & 100 & 57 & 73 & 100 & 100 & 100 & 84 & 100 & 91 \\
\hline D197 & 0 & 0 & 0 & 0 & 0 & 0 & 73 & 65 & 69 & 100 & 100 & 100 \\
\hline D24 & 21 & 100 & 34 & 47 & 100 & 64 & 65 & 73 & 69 & 100 & 100 & 100 \\
\hline Average & 4 & 21 & 7 & 57 & 64 & 56 & 86 & 86 & 86 & 95 & 94 & 94 \\
\hline
\end{tabular}

Table 4: Precision, Recall, and F1-score for shape-texture feature according to each durian species and classifiers

\begin{tabular}{|c|c|c|c|c|c|c|c|c|c|c|c|c|}
\hline \multirow{2}{*}{$\begin{array}{l}\text { Species / } \\
\text { Classifier }\end{array}$} & \multicolumn{3}{|c|}{ SVM (\%) } & \multicolumn{3}{|c|}{ LR (\%) } & \multicolumn{3}{|c|}{ GNB (\%) } & \multicolumn{3}{|c|}{ LDA (\%) } \\
\hline & $\mathbf{P}$ & $\mathbf{R}$ & F1 & $\mathbf{P}$ & $\mathbf{P}$ & $\mathbf{R}$ & F1 & $\mathbf{P}$ & $\mathbf{P}$ & $\mathbf{R}$ & F1 & $\mathbf{P}$ \\
\hline D101 & 100 & 84 & 91 & 56 & 100 & 72 & 100 & 100 & 100 & 100 & 100 & 100 \\
\hline D160 & 88 & 100 & 93 & 100 & 29 & 44 & 100 & 100 & 100 & 100 & 100 & 100 \\
\hline D197 & 100 & 100 & 100 & 100 & 100 & 100 & 100 & 100 & 100 & 100 & 100 & 100 \\
\hline D24 & 100 & 100 & 100 & 100 & 100 & 100 & 100 & 100 & 100 & 100 & 100 & 100 \\
\hline Average & 96 & 96 & 96 & 88 & 79 & 76 & 100 & 100 & 100 & 100 & 100 & 100 \\
\hline
\end{tabular}

\subsection{Classification Performance based on Fruits-360 Image Dataset}

For this particular experiment, larger dataset is used in comparison to the previous experiment. Initially the proposed shape-only descriptor was constructed to represent durian images. We tested the algorithm on 40 durian images in [29] and later expanded the collection to 240 images as reported in Section 5.1. We are performing this experiment to observe the ability of the proposed multiple feature descriptor in analysing other types of fruit. The Fruits-360 image dataset contains 42337 of fruit images with 64 fruit categories. For each category, the fruits are in various colour, shape, texture, size, orientation, and position. This dataset also contain different varieties of the same fruit which are treated as belonging to different classes (for example, for apple alone, there are 10 classes). A good feature extraction method and classifier should be able to differentiate these apple varieties. We compared the performance of the proposed features using different classifiers; Support Vector Machine (SVM), Logistic Regression (LR), Gaussian Naïve Bayesian (GNB), and Linear Discriminant Analysis (LDA). 70\% of the images are used for training (29636 images) and $30 \%$ of the images are used for testing (12701 images). Each predictor is trained using 10-fold cross validation. Several performance measurements are captured such as the accuracy $(A)$, precision $(P)$, recall $(R)$ and F1- 
score (F1). The experimental results obtained for the respective shape-only feature, texture-only feature, and feature fusion is tabulated in Table 5 .

From Table 5, it can be observed that the proposed feature fusion method confirmed its validity in providing good recognition performance in comparison to the other two methods. The proposed feature fusion method able to obtain the highest accuracy, precision, recall, and F1-score when using LR, GNB, and LDA classifiers. It is however a slightly different findings obtained by SVM classifier where precision, recall, and F1-score of shape-texture descriptor is similar to that of shape-only descriptor. In comparison to the previous experiment in Section 5.1 where shape-only feature performed better for the durian image dataset rather than texture-only feature, we can observe that texture feature is more prominent for the fruits-360 image dataset. This is probably due to the reason that this image dataset consists of more fruit varieties where the texture of one fruit to another is very different. Nevertheless, these two features performed much better when combined. This is due to the different features being extracted by the respective proposed features which allow for the images to be accurately represented. Area, perimeter, and circularity of the fruits as well as the small-scale texture appearance of the fruit's texture allow for the important information to be represented. All of these characteristics that actually translated the proposed shape-texture method to become effective.

Table 5: Accuracy, Precision, Recall, and F1-score of various features according to each classifier

\begin{tabular}{|l|c|c|c|c|c|c|c|c|c|c|c|c|c|c|c|c|}
\hline $\begin{array}{l}\text { Method / } \\
\text { Classifier }\end{array}$ & \multicolumn{4}{|c|}{ SVM (\%) } & \multicolumn{4}{|c|}{ LR (\%) } & \multicolumn{4}{c|}{ GNB (\%) } & \multicolumn{4}{c|}{ LDA (\%) } \\
\hline $\begin{array}{l}\text { Shape-only } \\
{[\mathbf{2 9 ]}}\end{array}$ & 25.03 & 19 & 25 & 19 & 17 & 12 & 17 & 11 & 23.13 & 18 & 23 & 16 & 19.26 & 15 & 10 & 15 \\
\hline $\begin{array}{l}\text { Texture- } \\
\text { only }\end{array}$ & 2.33 & 0 & 2 & 0 & 48.88 & 52 & 49 & 44 & 50.7 & 51 & 51 & 48 & 59.09 & 6 & 59 & 59 \\
\hline $\begin{array}{l}\text { Shape- } \\
\text { Texture } \\
\text { (proposed) }\end{array}$ & 25.05 & 19 & 25 & 19 & 61.89 & 64 & 62 & 60 & 63.91 & 66 & 64 & 63 & 72.38 & 73 & 72 & 72 \\
\hline
\end{tabular}

In terms of classification model, it is shown that LDA performed the best compared to the other three classification models. Since the Fruits-360 dataset is quite large with 64 categories, we have only detailed out the precision, recall, and F1-score according to fruit category for LDA classifier. The results can be observed in Table 6 below.

Table 6: Precision, Recall, and F1-score according to fruit categories based on LDA classifier

\begin{tabular}{|l|c|c|c|c|c|c|c|c|c|}
\hline \multirow{2}{*}{$\begin{array}{c}\text { Category / } \\
\text { Method }\end{array}$} & \multicolumn{3}{c|}{ Shape-only [27] } & \multicolumn{3}{c|}{ Texture-only } & \multicolumn{3}{c|}{ Shape-Texture (Proposed) } \\
\cline { 2 - 11 } & P (\%) & R (\%) & F1 (\%) & P (\%) & R (\%) & F1 (\%) & P (\%) & R (\%) & F1 (\%) \\
\hline Apple Braeburn & 2 & 1 & 1 & 54 & 50 & 52 & 60 & 51 & 56 \\
\hline Apple Golden 1 & 10 & 7 & 8 & 65 & 55 & 60 & 65 & 88 & 75 \\
\hline Apple Golden 2 & 23 & 28 & 25 & 58 & 73 & 65 & 79 & 95 & 86 \\
\hline Apply Golden 3 & 26 & 18 & 21 & 56 & 74 & 64 & 67 & 82 & 74 \\
\hline $\begin{array}{l}\text { Apple Granny } \\
\text { Smith }\end{array}$ & 37 & 50 & 43 & 61 & 74 & 67 & 78 & 93 & 85 \\
\hline Apply Red 1 & 3 & 2 & 2 & 35 & 28 & 31 & 52 & 51 & 52 \\
\hline Apple Red 2 & 14 & 9 & 11 & 28 & 36 & 32 & 39 & 47 & 43 \\
\hline Apple Red 3 & 13 & 18 & 15 & 23 & 48 & 31 & 38 & 61 & 47 \\
\hline $\begin{array}{l}\text { Apple Red } \\
\text { Delicious }\end{array}$ & 6 & 1 & 1 & 72 & 79 & 75 & 79 & 77 & 78 \\
\hline $\begin{array}{l}\text { Apple Red } \\
\text { Yellow }\end{array}$ & 0 & 0 & 0 & 60 & 40 & 48 & 51 & 31 & 38 \\
\hline Apricot & 0 & 0 & 0 & 63 & 84 & 72 & 82 & 86 & 84 \\
\hline Avocado & 8 & 2 & 4 & 67 & 45 & 54 & 66 & 68 & 67 \\
\hline Avocado ripe & 24 & 39 & 30 & 98 & 76 & 86 & 97 & 80 & 88 \\
\hline Banana & 10 & 14 & 12 & 57 & 56 & 56 & 73 & 59 & 65 \\
\hline Banana Red & 0 & 0 & 0 & 57 & 34 & 43 & 70 & 58 & 64 \\
\hline
\end{tabular}




\begin{tabular}{|c|c|c|c|c|c|c|c|c|c|}
\hline Cactus fruit & 3 & 3 & 3 & 62 & 73 & 67 & 69 & 76 & 72 \\
\hline Cantaloupe 1 & 49 & 100 & 66 & 79 & 94 & 86 & 99 & 100 & 9 \\
\hline Cantaloupe 2 & 23 & 58 & 33 & 80 & 56 & 66 & 99 & 97 & 98 \\
\hline Carambula & 4 & 4 & 4 & 57 & 61 & 59 & 80 & 77 & 78 \\
\hline Cherry 1 & 11 & 30 & 17 & 55 & 75 & 63 & 61 & 83 & 70 \\
\hline Cherry 2 & 9 & 48 & 16 & 58 & 43 & 50 & 67 & 50 & 57 \\
\hline Cherry Rainier & 4 & 7 & 5 & 47 & 47 & 47 & 52 & 34 & 41 \\
\hline Clementine & 22 & 21 & 21 & 19 & 11 & 13 & 70 & 55 & 62 \\
\hline Cocos & 36 & 4 & 7 & 70 & 83 & 76 & 95 & 96 & 95 \\
\hline Dates & 4 & 5 & 4 & 95 & 72 & 82 & 95 & 77 & 85 \\
\hline Granadilla & 20 & 22 & 21 & 58 & 62 & 60 & 73 & 75 & 74 \\
\hline Grape Pink & 0 & 0 & 0 & 38 & 34 & 36 & 55 & 64 & 59 \\
\hline Grape White & 69 & 85 & 77 & 73 & 81 & 77 & 97 & 97 & 97 \\
\hline Grape White 2 & 0 & 0 & 0 & 90 & 99 & 95 & 100 & 100 & 100 \\
\hline Grapefruit Pink & 29 & 47 & 36 & 61 & 51 & 56 & 83 & 88 & 85 \\
\hline $\begin{array}{l}\text { Grapefruit } \\
\text { White }\end{array}$ & 27 & 50 & 35 & 74 & 85 & 7 & 69 & 96 & 81 \\
\hline Guava & 18 & 46 & 26 & 42 & 66 & 51 & 59 & 79 & 68 \\
\hline Huckleberry & 4 & 4 & 4 & 42 & 52 & 46 & 53 & 67 & 59 \\
\hline Kaki & 0 & 0 & 0 & 51 & 49 & 50 & 72 & 72 & 72 \\
\hline Kiwi & 22 & 11 & 14 & 58 & 50 & 54 & 85 & 80 & 83 \\
\hline Kumquats & 23 & 51 & 32 & 62 & 72 & 67 & 78 & 75 & 77 \\
\hline Lemon & 4 & 3 & 3 & 48 & 44 & 46 & 73 & 67 & 70 \\
\hline Lemon Meyer & 9 & 1 & 2 & 68 & 69 & 69 & 83 & 88 & 86 \\
\hline Limes & 12 & 2 & 3 & 61 & 63 & 62 & 72 & 74 & 73 \\
\hline Litchi & 17 & 33 & 22 & 88 & 92 & 90 & 98 & 94 & 96 \\
\hline Mandarine & 16 & 7 & 10 & 41 & 40 & 41 & 72 & 71 & 71 \\
\hline Mango & 0 & 0 & 0 & 60 & 76 & 67 & 74 & 79 & 76 \\
\hline Maracuja & 0 & 0 & 0 & 52 & 25 & 34 & 57 & 65 & 61 \\
\hline Nectarine & 0 & 0 & 0 & 36 & 39 & 37 & 39 & 30 & 34 \\
\hline Orange & 37 & 77 & 50 & 81 & 87 & 84 & 95 & 98 & 96 \\
\hline Papaya & 0 & 0 & 0 & 64 & 41 & 50 & 85 & 81 & 83 \\
\hline Passion Fruit & 20 & 14 & 17 & 35 & 38 & 37 & 50 & 57 & 53 \\
\hline Peach & 0 & 0 & 0 & 38 & 45 & 41 & 39 & 2 & 33 \\
\hline Peach Flat & 0 & 0 & 0 & 50 & 37 & 43 & 64 & 50 & 56 \\
\hline Pear & 19 & 23 & 21 & 79 & 61 & 69 & 96 & 74 & 84 \\
\hline Pear Abate & 7 & 3 & 5 & 85 & 76 & 80 & 96 & 72 & 82 \\
\hline Pear Monster & 15 & 9 & 12 & 68 & 43 & 53 & 80 & 73 & 77 \\
\hline Pear Williams & 13 & 11 & 12 & 40 & 28 & 33 & 75 & 46 & 57 \\
\hline Pepino & 9 & 8 & 9 & 74 & 70 & 72 & 87 & 76 & 81 \\
\hline Pineapple & 36 & 20 & 25 & 79 & 84 & 81 & 93 & 89 & 91 \\
\hline Pitahaya Red & 34 & 49 & 40 & 49 & 60 & 54 & 67 & 87 & 76 \\
\hline Plum & 16 & 16 & 16 & 39 & 50 & 44 & 50 & 72 & 59 \\
\hline Pomegranate & 25 & 0 & 1 & 21 & 23 & 22 & 29 & 38 & 32 \\
\hline Quince & 27 & 23 & 25 & 71 & 71 & 71 & 83 & 83 & 83 \\
\hline Raspberry & 23 & 48 & 31 & 86 & 92 & 89 & 93 & 97 & 5 \\
\hline Salak & 49 & 82 & 61 & 74 & 81 & 77 & 77 & 82 & 79 \\
\hline Strawberry & 18 & 16 & 17 & 79 & 65 & 71 & 95 & 68 & 79 \\
\hline Tamarillo & 3 & 7 & 5 & 96 & 86 & 90 & 94 & 90 & 92 \\
\hline Tangelo & 19 & 2 & 3 & 53 & 42 & 47 & 80 & 78 & 79 \\
\hline Average & 15 & 19 & 15 & 60 & 59 & 59 & 73 & 72 & 72 \\
\hline
\end{tabular}

Based on the results tabulated in Table 6, it can be perceived that the descriptor of multiple features performed more consistently across different fruit categories. It is also able to achieve highest average performance for all three different evaluation measurements which is $73 \%$ precision, $72 \%$ recall, and $72 \% \mathrm{~F} 1$-score. 
Based on the results obtained in experiments 1 and 2, it can be concluded that the proposed feature fusion method showed to be the most effective in comparison to the individual features. Among the trained classifiers, LDA is said to be the most suitable classification model for this work.

\subsection{CONCLUSIONS AND FUTURE WORKS}

Fruit recognition framework specifically for durian needs further investigations. This is because this 'king of fruits' have few similar characteristics between different species where the skin have almost the same colour from green to yellowish brown with slightly different shape of thorns and it is hard to differentiate them with the current methods. Inspired by these challenges, this research proposed a new content-based descriptor based on shape and texture for durian image representation. For this purpose, shape information is represented based on shape signature while texture information is extracted based on Local Binary Pattern (LBP) descriptor. These features are then fed into few classifiers for recognition purposes. Experiments conducted on two image dataset (240 durian images and 42337 fruit images) have shown that image descriptor of multiple features (shape-texture) performed better in comparison to shape-only [29] and texture-only descriptors. It has also been observed that LDA classifier performed the best in comparison to LR, SVM, and GNB. The obtained results are $100 \%$ for accuracy, precision, recall, and F1-score for durian dataset, and $72.38 \%$ accuracy, $73 \%$ precision, $72 \%$ recall, and $71 \% \mathrm{~F} 1$-score for fruits dataset.

For future works, few other classifiers such as Random Forest and Neural Network may be considered to further improve the method. Apart from that, some of the different durian species may have almost similar base, shape of spines, and shape of fruit. Hence, colour feature could also be used as part of image representation to differentiate the various durian species.

\section{ACKNOWLEDGEMENT}

This work is supported by Universiti Putra Malaysia under the Putra Grant project code GP/2018/9644500.

\section{REFERENCES}

[1] C. Ding, J. Choi, D. Tao, and L. S. Davis, "Multi-directional multi-level dual-cross patterns for robust face recognition". IEEE Transactions on Pattern Analysis and Machine Intelligence, Vol. 38, No. 3, pp. 518-531, 2016.

[2] C. Ding and D. Tao, "Robust face recognition via multimodal deep face representation". IEEE Transactions on Multimedia, Vol. 17, No. 11, pp. 2049-2058, 2015.

[3] A. Boles and P. Rad, "Voice biometrics: Deep learning-based voiceprint authentication system", in Proceedings of the $12^{\text {th }}$ System of Systems Engineering Conference (SoSE), 2017, pp. 1-6. IEEE.

[4] P. Kumar, R. Saini, P. P. Roy, and U. Pal, "A lexicon-free approach for 3D handwriting recognition using classifier combination”. Pattern Recognition Letters, Vol. 103, pp. 1-7 2018.

[5] Kahou, Samira Ebrahimi, Xavier Bouthillier, Pascal Lamblin, Caglar Gulcehre, Vincent Michalski, Kishore Konda, Sébastien Jean et al., "Emonets: Multimodal deep learning approaches for emotion recognition in video". Journal on Multimodal User Interfaces, Vol. 10, No. 2, pp. 99-111, 2016.

[6] S. Escalera, V. Athitsos, and I. Guyon, "Challenges in multi-modal gesture recognition", in Gesture Recognition, pp. 1-60, 2017. Springer, Cham.

[7] E. g. Llano, M. s. G. Vázquez, J. M. C. Vargas, L. M. Z. Fuentes, and A. A. R. Acosta, “Optimized robust multi-sensor scheme for simultaneous video and image iris recognition". Pattern Recognition Letters, Vol. 101, pp. 44-51, 2018.

[8] S. Idris, Durio of Malaysia. Kuala Lumpur, Malaysia: Malaysian Agricultural Research and Development Institute, MARDI, 2011. 
[9] S. Ketsa, "Durian - Durio zibethinus". Exotic Fruits, pp. 169-180, 2018.

[10] M. R. Mustaffa, F. Ahmad, and S. C. Doraisamy, "Multi-Resolution Joint Auto Correlograms for ContentBased Image Retrieval”. Advanced Science Letters, Vol. 23, No. 6, pp. 5370-5374, 2017.

[11] S. Karamizadeh, S. M. Abdullah, M. Zamani, and A. Kherikhah, "Pattern recognition techniques: studies on appropriate classifications", in Advanced Computer and Communication Engineering Technology, 2015, pp. 791-799. Springer, Cham.

[12] W. C. Seng and S. H. Mirisaee, "A new method for fruit recognition system", in Proceedings of the International Conference on Electrical Engineering and Informatics, 2009, pp. 130-134.

[13] Official Portal of Department of Agriculture of Ministry Agriculture \& Agro- Based Industry. (2017, April 22). Retrieved June 3, 2017, from http://pvpbkkt.doa.gov.my/

[14] N. S. Ibrahim, Z. Abu Bakar, N. Abd. Rahman, "Characteristics classification of durian varieties images for knowledge representation by using conceptual graph", in Information Retrieval Technology: Proceedings of the 10th Asia Information Retrieval Societies Conference (AIRS 2014), Kuching, Malaysia, December 3-5, 2014, A. Jaafar, N. Mohamad Ali, S. A. Mohd Noah, A. F. Smeaton, P. Bruza, Z. Abu Bakar, N. Jamil, and T. M. Tengku Sembok, Eds. Heidelberg: Springer International Publishing, pp. 124-135.

[15] Y. Song, C. A. Glasbey, G. W. Horgan, G. Polder, J. A. Dieleman, and G. W. A. M. Van der Heijden, "Automatic fruit recognition and counting from multiple images". Biosystems Engineering, Vol. 118, pp. 203-215, 2014.

[16] P. Ninawe and M. S. Pandey, "A completion on fruit recognition system using k-nearest neighbours algorithm”. International Journal of Advanced Research in Computer Engineering \& Technology (IJARCET), Vol. 3, No. 7, pp. 2352-2356, 2014.

[17] S. Jana and R. Parekh, "Shape-based Fruit Recognition and Classification", in Proceedings of the International Conference on Computational Intelligence, Communications, and Business Analytics, 2017, pp. 184-196. Springer, Singapore.

[18] P. A. Macanhã, D. M. Eler, R. E. Garcia, and W. E. M. Junior, "Handwritten Feature Descriptor Methods Applied to Fruit Classification”, in Information Technology-New Generations, 2018, pp. 699-705. Springer, Cham.

[19] I. Sa, Z. Ge, F. Dayoub, B. Upcroft, T. Perez, and C. McCool, "Deepfruits: A fruit detection system using deep neural networks”. Sensors, Vol. 16, No. 8, pp. 1222, 2016.

[20] G. Zeng, "Fruit and vegetables classification system using image saliency and convolutional neural network", in IEEE $3^{\text {rd }}$ Information Technology and Mechatronics Engineering Conference (ITOEC), 2017, pp. 613617. IEEE.

[21] L. Hou, Q. Wu, Q. Sun, H. Yang, P. Li, "Fruit recognition based on convolution neural network", in 2016 12th International Conference on Natural Computation, Fuzzy Systems and Knowledge Discovery (ICNCFSKD), 2016, pp. 18-22. IEEE.

[22] G. Gatica, S. Best, J. Ceroni, and G. Lefranc, "Olive fruits recognition using neural networks". Procedia Computer Science, Vol. 17, pp. 412-419, 2013.

[23] M. Khanmohammadi, F. Karami, A. Mir-Marqués, A. B. Garmarudi, S. Garrigues, and M. De La Guardia, "Classification of persimmon fruit origin by near infrared spectrometry and least squares-support vector machines”. Journal of Food Engineering, Vol. 142, pp. 17-22, 2014.

[24] A. Nasirahmadi and S. H. M. Ashtiani, "Bag-of-Feature model for sweet and bitter almond classification". Biosystems Engineering, Vol. 156, pp. 51-60, 2017.

[25] L. Xu and J. Lv, "Recognition method for apple fruit based on SUSAN and PCNN". Multimedia Tools and Applications, Vol. 77, No. 6, pp. 7205-7219, 2018. 
[26] L. F. S Pereira, S. Barbon, N. A. Valous, and D. F. Barbin, "Predicting the ripening of papaya fruit with digital imaging and random forests”. Computers and Electronics in Agriculture, Vol. 145, pp. 76-82, 2018.

[27] F. Pensiri and P. Visusak, "Durian cultivar recognition using discriminant function", in Proceedings of the $20172^{\text {nd }}$ International Conference on Information Technology (INCIT), 2-3 November 2017, Nakhonpathom, Thailand, 2017, pp. 1-5. IEEE.

[28] R. Phoophuangpairoj, "Computerized unripe and ripe durian striking sound recognition using syllable-based HMMs”. Applied Mechanics and Materials, Vol. 446, pp. 927-935, 2014.

[29] X. Y. Nyon, M. R. Mustaffa, L. N. Abdullah, and N. A. Nasharuddin, "Durian species recognition system based on global shape representations and k-nearest neighbours", in Proceedings of the 2018 Fourth International Conference on Information Retrieval and Knowledge Management (CAMP'18), 26-28 March 2018, Kota Kinabalu, Malaysia. IEEE. (In press).

[30] C. Cortes and V. Vapnik, "Support-vector networks". Machine learning, Vol. 20, No. 3, pp. 273-297, 1995.

[31] H.-Y. Huang and C.-J. Lin, "Linear and kernel classification: When to use_which?", in Proceedings of the 2016 SIAM International Conference on_Data Mining (SIAM), 2016, pp. 216-224.

[32] D. W. Hosmer Jr., S. Lemeshow, and R. X. Sturdivant, Applied logistic regression, (Vol. 398). John Wiley \& Sons, 2013.

[33] G. H. John, and P. Langley, "Estimating continuous distributions in Bayesian classifiers", in Proceedings of the Eleventh conference on Uncertainty in artificial intelligence, pp. 338-345, Morgan Kaufmann Publishers Inc.

[34] S. Mika, G. Ratsch, J. Weston, B. Scholkopf, and K. R. Mullers, "Fisher discriminant analysis with kernels", in Neural networks for signal processing IX, 1999. Proceedings of the 1999 IEEE signal processing society workshop, 1999, pp. 41-48. IEEE.

[35] P. D. Leedy and J. E. Ormrod. Practical Research: Planning and Design, 10th ed., Harlow: Pearson Education Limited, 2014.

[36] Horea Muresan and Mihai Oltean, Fruit recognition from images using deep learning, Technical Report, Babes-Bolyai University, 2017. 